\title{
dbNSFP v4: a comprehensive database of transcript-specific functional predictions and annotations for human nonsynonymous and splice-site SNVs
}

\author{
Xiaoming Liu ${ }^{1 *} \mathbb{D}$, Chang $\mathrm{Li}^{1}$, Chengcheng Mou${ }^{2}$, Yibo Dong ${ }^{1}$ and Yicheng $\mathrm{Tu}^{2}$
}

\begin{abstract}
Whole exome sequencing has been increasingly used in human disease studies. Prioritization based on appropriate functional annotations has been used as an indispensable step to select candidate variants. Here we present the latest updates to dbNSFP (version 4.1), a database designed to facilitate this step by providing deleteriousness prediction and functional annotation for all potential nonsynonymous and splice-site SNVs (a total of 84,013,093) in the human genome. The current version compiled 36 deleteriousness prediction scores, including 12 transcriptspecific scores, and other variant and gene-level functional annotations. The database is available at http://database. liulab.science/dbNSFP with a downloadable version and a web-service.
\end{abstract}

Keywords: Whole exome sequencing, Database, Nonsynonymous SNV, Deleteriousness prediction, Functional annotation

\section{Background}

Whole-exome sequencing (WES) and whole-genome sequencing (WGS) have been increasingly used in human disease studies in the research and clinical setting [1-3]. As a result, we witness a tsunami of DNA sequence data from both healthy individuals and those with Mendelian or complex diseases. Identifying variants that cause diseases or are associated with disease risks from a large number of DNA variants identified in sequencing requires an excessive amount of time and effort. To accomplish this daunting task, investigators have relied on functional annotations to filter or prioritize variants based on our current knowledge or prediction models. In more detail, functional annotations can be separated into general annotation and functional prediction: the

\footnotetext{
* Correspondence: xiaomingliu@usf.edu

'USF Genomics \& College of Public Health, University of South Florida, Tampa, FL, USA

Full list of author information is available at the end of the article
}

former provides qualitative or descriptive annotation of a variant indirectly related to its potential function, such as whether the variant is a nonsynonymous SNV; the latter typically provides direct quantitative or yes-or-no deleteriousness prediction of the variant based on a statistical model. Fast and comprehensive functional annotation tools will become even more critical in the near future because of three intertwined ongoing trends: the decreasing cost of DNA sequencing, the development and practice of precision medicine [4], and the adaptation of WES and WGS in small labs [5].

There have been several annotation tools available for large-scale DNA sequence data, such as UCSC Genome Browser's Variant Annotation Integrator [6], Ensembl's Variant Effect Predictor (VEP) [7], ANNOVAR [8], and SnpEff [9]. Most of these focused on general annotations based on given gene models. Although gene-model based annotations are handy, there are other important functional annotation resources used by medical 
geneticists and genetic epidemiologists, including functional prediction of variants, conservation information, predicted promoters, enhancers, and epigenomic markers, among others. Another challenge faced by the investigators is that different gene-model-based annotation tools all have their advantages and disadvantages, and the results sometimes do not agree with each other [10]. Therefore, it has been suggested to obtain annotation from tools across multiple databases for a complete interpretation of the variants. Previously, we developed dbNSFP version 1 [11], 2 [12], and 3 [13] to provide a "one-stop-shop" for functional annotations for nonsynonymous SNVs (nsSNVs) and splice site SNVs (ssSNVs), top candidate variant types for Mendelian diseases. It collected all possible nsSNVs and ssSNVs based on human reference sequences and multiple deleteriousness predictions and annotations for each SNV.

Here we report the major updates of dbNSFP since version 3.0 to the current version 4.1. The core SNVs have been rebuilt based on human reference sequence version hg38 and GENCODE version 29 [14]. Compared to version 3.0 [13], dbNSFP v4.1 added 18 deleteriousness prediction scores (BayesDel_addAF and BayesDel_noAF [15], CADD_hg19 [16], ClinPred [17], DEOGEN2 [18], Eigen and Eigen PC [19], FATHMM-XF [20], GenoCanyon [21], LINSIGHT [22], LIST-S2 [23], M-CAP [24], MPC [25], MutPred [26], MVP [27], PrimateAI [28], REVEL [29], SIFT4G [30]), one score for loss of function prediction (ALoFT [31]), and three conservation scores (phyloP17way_primate [32], phastCons17way_primate [33],
bStatistic [34]), making the total number of prediction scores to 46 (Additional file 1: Table S1). Many other functional annotation resources have been added or updated. In addition to the previously supported query of two attached databases, dbscSNV [35] and SPIDEX [36], for predicting splice interrupting SNVs, the companion query program for the downloadable version added support for querying SpliceAI, a third-party database for predicting splice site gain and loss [37], and dbMTS, a comprehensive database for microRNA target site SNVs and their functional predictions [38]. More importantly, much effort has been made to increase further the usability of the functional annotations, including (1) making functional predictions transcript-specific whenever possible, (2) providing transcript annotations to help to choose appropriate transcript from multiple isoforms for each gene, (3) providing HGVS (Human Genome Variation Society) c. and p. presentations of the SNVs to facilitate the query of candidate mutations reported in medical genetics literatures, and (4) providing graphic user interface for querying downloadable version as well as webservice for researchers with minimum bioinformatics training.

\section{Construction and content}

We rebuilt the list of all potential nonsynonymous and splice-site SNVs based on the GENCODE gene model version 29 (Ensembl version 94) with human reference sequence GRCh38. Only transcripts with complete proteincoding annotations were included. A total of 81,782,923

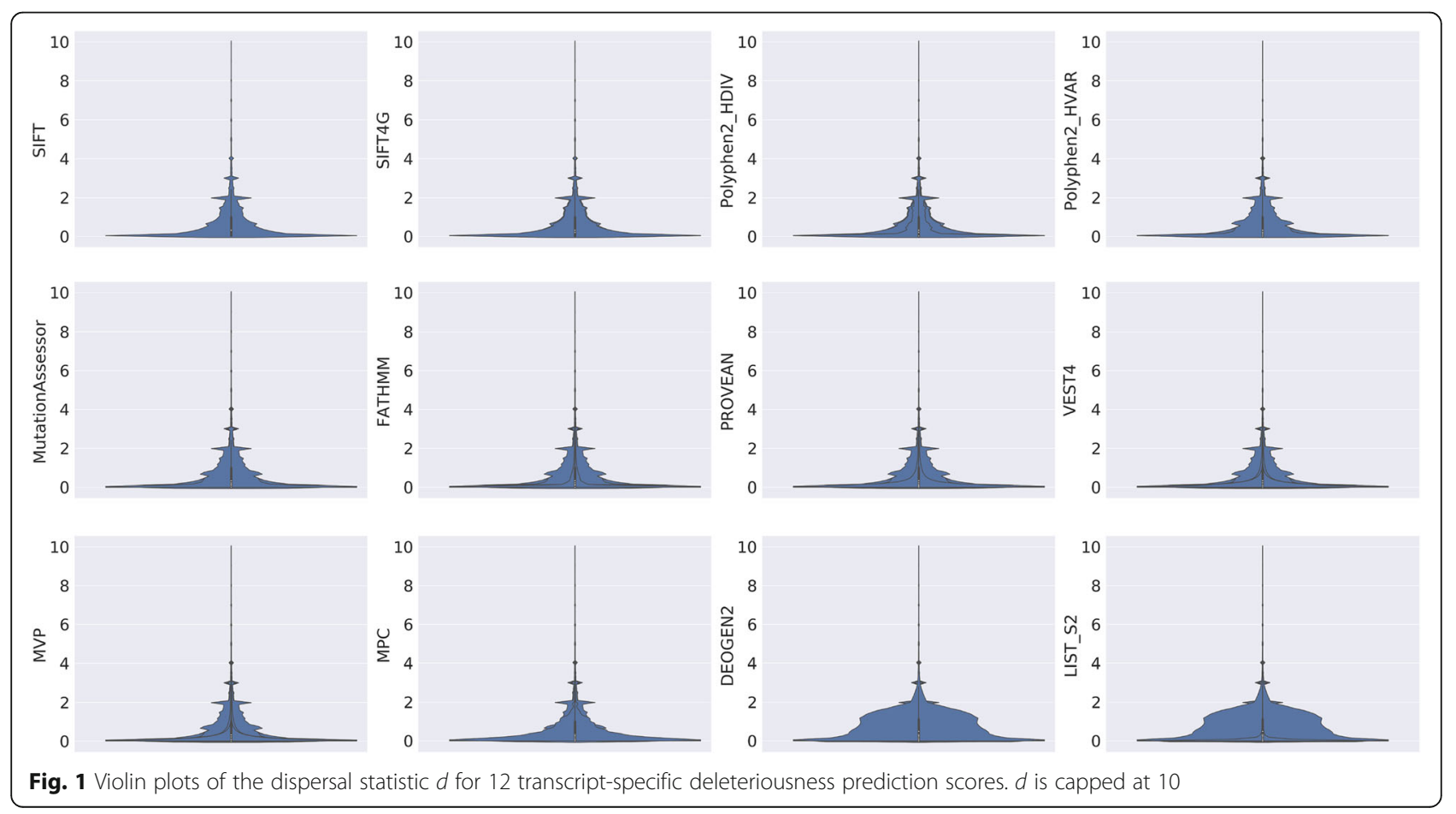


nsSNVs and 2,230,170 ssSNVs were collected in the database (Additional file 2: Table S2). The corresponding chromosomal positions of the SNVs based on human reference sequences hg19 and hg18 were obtained via the liftover tool [39] (Additional file 2: Table S2). Accurate protein ID mapping between GENCODE/Ensembl and Uniprot [40] was obtained via a comprehensive protein sequence matching between all the proteins in GENCODE/ Ensembl and those of the Uniprot database. To facilitate the choice of the appropriate transcript(s) for each gene, we collected transcript quality measures including APPR
IS [41], transcript support level (TSL), GENCODE Basic, and Ensembl canonical transcripts were obtained from the Ensembl Biomart [42] and Variant Effect Predictor (VEP). HGVS c. and p. presentations by ANNOVAR, snpEff, and VEP for each nsSNV and ssSNV were obtained via the WGSA (WGS Annotator) pipeline [43]. As a core content of dbNSFP, 36 deleteriousness prediction scores, nine conservation scores, and one loss of function score for each nsSNV or ssSNV were compiled (see Additional file 1: Table S1 for a summary). Among them, 13 scores are transcript-specific (ALoFT, DEOGEN2, FATHMM [44],

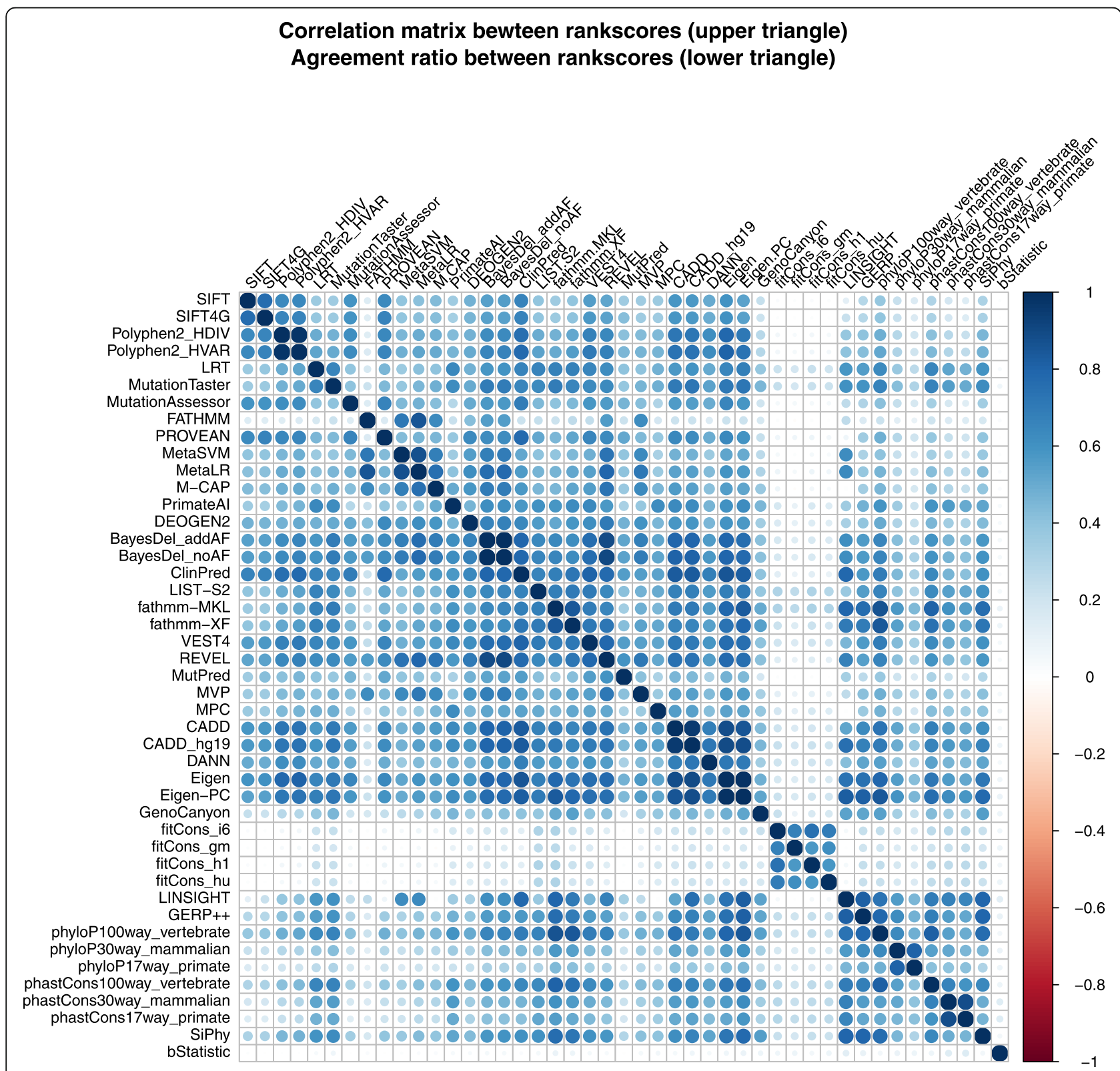

Fig. 2 Pearson's correlation coefficients of rank scores (upper triangle) and agreement ratio of binary predictions (lower triangle) between pairs of deleteriousness prediction scores or conservation scores 


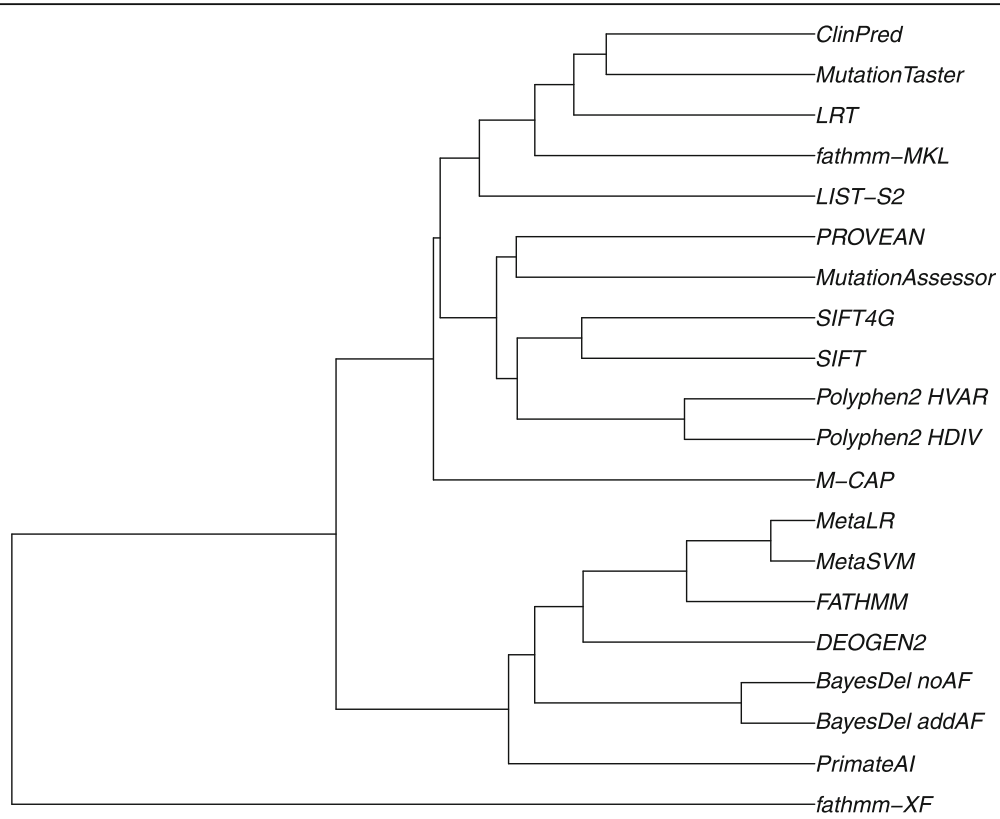

Fig. 3 UPGMA dendrogram of the deleteriousness prediction scores and conservation scores

LIST-S2, MPC, MutationAssessor [45], MVP, Polyphen2 HDIV and Polyphen2 HVAR [46], PROVEAN [47], SIFT [48], SIFT4G, VEST4 [49]). The full list of annotation resources and the description of all columns in dbNSFP can be found at http://database.liulab.science/dbNSFP.

\section{Utility and discussion}

\section{Query utility}

dbNSFP v4.1 can be accessed as either a downloadable and standalone version, or as a web-service at http:// database.liulab.science/dbNSFP. The standalone version is suitable for a large-scale query, such as quickly identifying nsSNVs and ssSNVs from exome sequencing studies. As no internet connection is required, maximum speed and security can be achieved. The query can be conducted via the companion Java program, which supports both the command-line and graphic user interface (GUI). The query term can be either a genomic position (chromosome, position), an SNV (chromosome, position, reference allele, alternative allele), an amino acid (AA) change (chromosome, position, reference allele, alternative allele, reference AA, alternative AA), a dbSNP ID (rs number), an HGVS c. or p. presentation of a mutation, or a gene name or ID. The companion Java program also supports searching attached databases along with dbNSFP, including dbscSNV, SPIDEX, spliceAI, and dbMTS, which helps to identify candidate diseasecausing SNVs affecting splicing and miRNA binding.

The web-service, which is managed by Microsoft SQL Server 2017, is suitable for a small-scale query such as obtaining functional annotations for candidate SNVs. By submitting one or multiple genome coordinates (chromosome, position, reference allele, and alternate allele), users can easily retrieve all the annotation columns in dbNSFP. The output will be displayed on the web page and available as a downloadable TAB-delimited text file for further filtering.

\section{Comparison of prediction scores}

dbNSFP is in a unique position for comparing different deleteriousness prediction scores and conservation scores across the whole exome. Among the 36 deleteriousness prediction scores, the average missing rate is 11\% (Additional file 2: Table S2). MVP has the lowest missing rate $(0.028 \%)$; three scores have missing rates > 20\%: ClinPred (21.7\%), MutationAssessor (22.2\%), LINSIGHT (97.7\%). The very high missing rate of LINSIG $\mathrm{HT}$ is due to that it was designed for noncoding variants. For the 9 conservation scores, the average missing rate is $0.6 \%$, with minimum $0.01 \%$ (phyloP100way_vertebrate and phastCons100way_vertebrate) and maximum 1.8\% (bStatistic) (Additional file 2: Table S2).

We first compare the dispersal of the scores for the same nsSNV affecting multiple transcripts, for the 12 transcript-specific deleteriousness prediction scores. In more details, for each nsSNV affecting more than one transcript, we calculate $d=\frac{\max -\min }{\text { ave }}$, where $\max , \min$, and ave are the maximum, minimum, and average of all transcript-specific scores. Of all the scores except FATHMM, there are sizable proportions of nsSNVs with a $d>2$, suggesting that choosing an appropriate transcript is essential for predicting the impact of the SNVs (Fig. 1). 
Then we compared the distribution of the scores. Because different score has a different scaling system, we create a rank score for each score so that it is comparable between scores [13]. The rank score has a scale 0 to 1 and represents the percentage of scores that are less damaging in dbNSFP, e.g., a rank score of 0.9 means the top $10 \%$ most damaging. We calculated the density distribution of the rank scores of 45 deleteriousness prediction scores and conservation scores (Additional file 3: Fig. S1, Additional file 2: Table S3). While most scores are in general evenly distributed, some scores are notably spiky and sparsely distributed, such as LRT [50], MutationTaster [51], GenoCanyon, phastCons100way_ vertebrate, and phastCons30way_mammalian, among others.

We also compared the correlation between scores. For the 45 deleteriousness prediction scores or conservation scores, we calculated Pearson's correlation coefficients $(r)$ of their rank scores (Fig. 2, Additional file 2: Table S4). About $43.4 \%$ of the correlations are strong $(>0.5)$, and $26.7 \%$ of the correlations are medium $(0.3-0.5)$. It is noticeable that the fitCons scores have a weak correlation with other scores, except between themselves. bStatistic has weak correlations with all other scores, suggesting that the strength of background selection it measured is quite different from other conservation scores. Using 1-r as a distance measure, we constructed a UPGMA (Unweighted Pair Group Method with Arithmetic Mean) dendrogram of the scores (Fig. 3). Interestingly, the ensemble scores or hybrid ensemble scores in dbNSFP form two separated clusters: cluster 1 includes CADD and CADD_hg19, ClinPred, BayesDel_addAF, BayesDel_noAF, and REVEL; cluster 2 includes MetaLR and MetaSVM [52], M-CAP, and DEOGEN2. This observation suggests that they captured different features of nsSNVs or weighted the features differently.

We also compared the agreement ratio of binary predictions by 20 deleteriousness prediction scores (Fig. 2, Additional file 2: Table S5). The median agreement ratio is 0.65 , which is reasonably high. Some of the highest agreement ratios are using the same training data, such as MetaLR and MetaSVM (0.96), BayesDel_addAF and BayesDel_noAF (0.94), Polyphen2_HDIV and Polyphen2_HVAR (0.88). On the other hand, some scores with similar algorithms do not have high agreement ratios: such as fathmm-XF and fathmm-MKL [53] (0.46). Fathmm-XF does not have a $>0.5$ agreement ratio with any other scores.

Finally, we compare the performance of the 45 deleteriousness prediction scores and conservation scores. We first collected a test set with true positive (TP) observations obtained from ClinVar between date 20200102 to 20200506 and with true negative (TN) observations obtained from gnomAD v2.1.1 hg38 in genomic locations

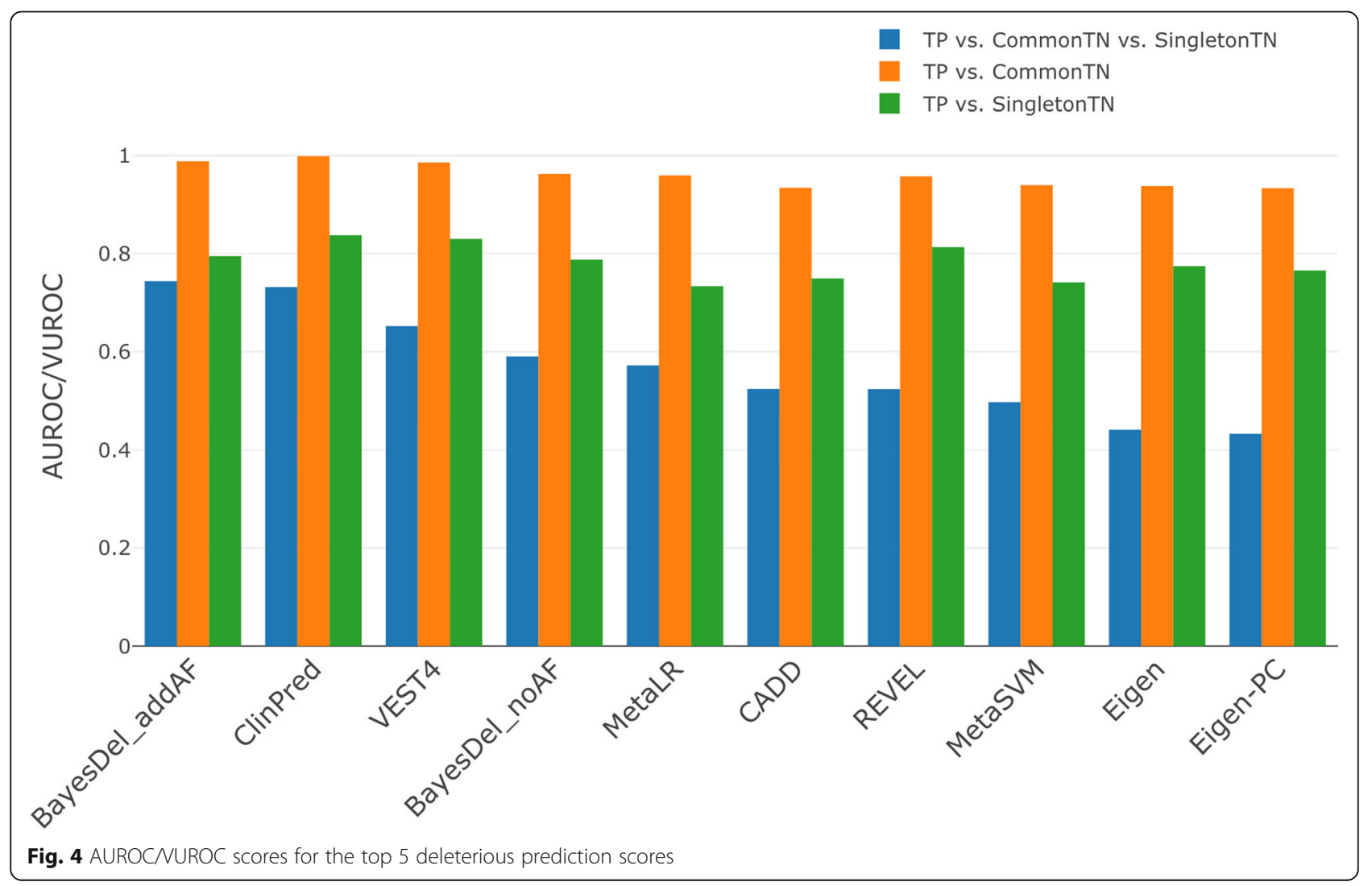


nearby the TP SNVs (Additional file 4: Supplementary methods). In total, we obtained 3113 missense SNVs as our TP group, and 55,914 missense SNVs as our TN group. Because the selection of TN controls is debatable as to whether to use very rare SNVs or to use common ones [54], we further divided our 55,914 TN SNVs into two groups. The first group (CommonTN; $n=1211$ ) contains SNVs with AF in gnomAD greater than $1 \%$. The second group (SingletonTN; $n=54,703$ ) contains singleton SNVs in gnomAD. We then calculated the area under the receiver operating characteristic (AUROC) for each score: one using TP vs. CommonTN and the other using TP vs. SingletonTN (Fig. 4, Additional file 2: Table S6). The top five performing scores for TP vs. CommonTN are ClinPred and BayesDel_addAF, VEST4, BayesDel_noAF, and MetaLR, while that for TP vs. SingletonTN are ClinPred, VEST4, REVEL, MutPred, and BayesDel_addAF. Interestingly, except for VEST4 and MutPred, all other scores are ensemble scores. As expected, the best AUROC for SingletonTN as control (0.8374) is substantially lower than it for CommonTN as control (0.999), highlighting the importance of future tools to provide better discriminatory power for rare benign SNVs.

As we expect that the SingletonTN group, in general, has a higher probability of being mildly deleterious than the CommonTN group, a score that can correctly distinguish the functional impact of CommonTN and SingletonTN should be more useful in the context of WES or WGS studies. Here, we extended the two-class AUROC to a 3-class volume under the ROC surface (VUROC) measure, which can simultaneously evaluate TP vs. SingletonTN vs. CommonTN. The resulting VUROC score represents the probability of correctly ranking the three test groups. A complete random guess (noninformative score) will have a VUROC of 0.167. Using a custom Python script, we calculated the VUROC for each of the 45 deleterious scores (Fig. 4, Additional file 2: Table S6). The top five performing scores are BayesDel_addAF $($ VUROC $=0.7443)$, ClinPred $($ VUROC $=0.7322)$, VEST4 $($ VUROC $=0.6525)$, BayesDel_noAF $($ VUROC $=0.5905)$, and MetaLR (VUROC $=0.5722$ ). Again, except for VEST4, all other scores are ensemble scores.

\section{Conclusions}

In conclusion, we present dbNSFP $\mathrm{v} 4$, a significant improvement over v3 over the past 4 years, as to supporting transcript-specific predictions and annotations, convenience to use (GUI support, joint-query of attached databases, and web-service), and double the number of deleteriousness prediction scores as to nsSNV. dbNSFP will continue to serve the community of medical geneticists as to providing comprehensive and easily-accessible tools for functional annotations and predictions for SNVs that cause amino acid changes and splicing changes.

\section{Supplementary Information}

The online version contains supplementary material available at https://doi. org/10.1186/s13073-020-00803-9.

Additional file 1: Table S1. A summary of functional prediction scores and conservation scores in dbNSFP v4.1.

Additional file 2: Table S2. Nonmissing counts of ssSNV, nsSNV and 45 scores per chromosome. Table S3. Density of rank scores based on 100 bins (bin size $=0.01$ ). Table S4. Pearson's correlation coefficients between rank scores. Table S5. Ratio of binary predictions' agreement between scores. Table S6. AUROCNUROC scores between TP testing set and different TN testing sets for the 45 deleterious prediction scores in dbNSFP $\vee 4.1$.

Additional file 3: Fig. S1. Density plots of rank scores of 45 deleteriousness prediction scores or conservation scores (bin size $=0.01$ ).

Additional file 4. Supplementary methods.

\section{Abbreviations}

dbNSFP: Database for nonsynonymous SNPs' functional predictions; SNV: Single nucleotide variant; WES: Whole-exome sequencing; WGS: Wholegenome sequencing; VEP: Variant Effect Predictor; nsSNV: Nonsynonymous SNV; ssSNV: Splice site SNV; HGVS: Human Genome Variation Society; WGSA: WGS Annotator; TSL: Transcript support level; GUI: Graphic user interface; AA: Amino acid; UPGMA: Unweighted Pair Group Method with Arithmetic Mean; AUROC: Area under the receiver operating characteristic; VUROC: Volume under the ROC surface

\section{Acknowledgements \\ The authors acknowledge the developers of the original annotation resources to share their data.}

\section{Authors' contributions}

X.L. designed the study, constructed the database, wrote the query program for the downloadable version, and attached databases. C.L. conducted analyses of the prediction scores. C.M., Y.D., and C.T. developed the webservice. X.L., C.L., and C.M. wrote the manuscript. All authors read and approved the final manuscript.

\section{Funding}

The research was supported by the National Human Genome Research Institute grant 1R03HG011075 to X.L.

\section{Availability of data and materials}

The web service of dbNSFP v4 can be found at http://database.liulab. science/dbNSFP. The downloadable version of dbNSFP $v 4$ can be found at http://database.liulab.science/dbNSFP and https://sites.google.com/site/ jpopgen/dbNSFP. All data sources and websites for downloading can be found in Additional file 1: Table S1. dbNSFP v4 is also available at https://zenodo.org/record/4323592\#.X9zPhNhKiHs and https://zenodo.org/ record/4329970\#.X9zPrdhKiHs.

Ethics approval and consent to participate Not applicable.

\section{Consent for publication}

Not applicable.

\section{Competing interests}

The authors declare that they have no competing interests.

\section{Author details}

${ }^{1}$ USF Genomics \& College of Public Health, University of South Florida, Tampa, FL, USA. ${ }^{2}$ Department of Computer Science and Engineering, College of Engineering, University of South Florida, Tampa, FL, USA. 
Received: 17 September 2020 Accepted: 9 November 2020 Published online: 02 December 2020

\section{References}

1. Bamshad MJ, Ng SB, Bigham AW, Tabor HK, Emond MJ, Nickerson DA, et al. Exome sequencing as a tool for Mendelian disease gene discovery. Nat Rev Genet. 2011;12:745-55

2. Lupski JR, Belmont JW, Boerwinkle E, Gibbs RA. Clan genomics and the complex architecture of human disease. Cell. 2011;147:32-43.

3. Goldstein DB, Allen A, Keebler J, Margulies EH, Petrou S, Petrovski S, et al. Sequencing studies in human genetics: design and interpretation. Nat Rev Genet. 2013;14:460-70.

4. Friedman $A A$, Letai $A$, Fisher DE, Flaherty KT. Precision medicine for cancer with next-generation functional diagnostics. Nat Rev Cancer. 2015;15:747-56.

5. Noor AM, Holmberg L, Gillett C, Grigoriadis A. Big data: the challenge for small research groups in the era of cancer genomics. Br J Cancer. 2015;113: 1405-12.

6. Hinrichs AS, Raney BJ, Speir ML, Rhead B, Casper J, Karolchik D, et al. UCSC data integrator and variant annotation integrator. Bioinformatics. 2016;32: $1430-2$

7. McLaren W, Gil L, Hunt SE, Riat HS, Ritchie GRS, Thormann A, et al. The Ensembl Variant Effect Predictor. Genome Biol. 2016;17:122.

8. Wang K, Li M, Hakonarson H. ANNOVAR: functional annotation of genetic variants from high-throughput sequencing data. Nucleic Acids Res. 2010;38: e164.

9. Cingolani $P$, Platts $A$, Wang $L L$, Coon $M$, Nguyen $T$, Wang $L$, et al. A program for annotating and predicting the effects of single nucleotide polymorphisms, SnpEff: SNPs in the genome of Drosophila melanogaster strain w1118; iso-2; iso-3. Fly (Austin). 2012;6:80-92.

10. McCarthy DJ, Humburg P, Kanapin A, Rivas MA, Gaulton K. The WGS500 Consortium, et al. choice of transcripts and software has a large effect on variant annotation. Genome Med. 2014;6:26

11. Liu X, Jian X, Boerwinkle E. dbNSFP: a lightweight database of human nonsynonymous SNPs and their functional predictions. Hum Mutat. 2011;32: 894-9.

12. Liu X, Jian X, Boerwinkle E. dbNSFP v2.0: a database of human nonsynonymous SNVs and their functional predictions and annotations. Hum Mutat. 2013;34:E2393-402.

13. Liu X, Wu C, Li C, Boerwinkle E. dbNSFP v3.0: a one-stop database of functional predictions and annotations for human nonsynonymous and splice-site SNVs. Hum Mutat. 2016:37:235-41.

14. Harrow J, Frankish A, Gonzalez JM, Tapanari E, Diekhans M, Kokocinski F, et al. GENCODE: the reference human genome annotation for the ENCODE project. Genome Res. 2012;22:1760-74.

15. Feng B-J. PERCH: a unified framework for disease gene prioritization. Hum Mutat. 2017;38:243-51.

16. Rentzsch P, Witten D, Cooper GM, Shendure J, Kircher M. CADD: predicting the deleteriousness of variants throughout the human genome. Nucleic Acids Res. 2019;47:D886-94.

17. Alirezaie N, Kernohan KD, Hartley T, Majewski J, Hocking TD. ClinPred: prediction tool to identify disease-relevant nonsynonymous singlenucleotide variants. Am J Hum Genet. 2018;103:474-83.

18. Raimondi D, Tanyalcin I, Ferté J, Gazzo A, Orlando G, Lenaerts T, et al. DEOGEN2: prediction and interactive visualization of single amino acid variant deleteriousness in human proteins. Nucleic Acids Res. 2017;45: W201-6.

19. Ionita-Laza I, McCallum K, Xu B, Buxbaum JD. A spectral approach integrating functional genomic annotations for coding and noncoding variants. Nat Genet. 2016;48:214-20.

20. Rogers MF, Shihab HA, Mort M, Cooper DN, Gaunt TR, Campbell C. FATH MM-XF: accurate prediction of pathogenic point mutations via extended features. Bioinformatics. 2018;34:511-3.

21. Lu Q, Hu Y, Sun J, Cheng Y, Cheung K-H, Zhao H. A statistical framework to predict functional noncoding regions in the human genome through integrated analysis of annotation data. Sci Rep. 2015:5:10576.

22. Huang Y-F, Gulko B, Siepel A. Fast, scalable prediction of deleterious noncoding variants from functional and population genomic data. Nat Genet. 2017;49:618-24

23. Malhis N, Jacobson M, Jones SJM, Gsponer J. LIST-S2: taxonomy based sorting of deleterious missense mutations across species. Nucleic Acids Res;
Available from: https://academic.oup.com/nar/advance-article/doi/10.1093/ nar/gkaa288/5827198. [cited 2020 Jun 20].

24. Jagadeesh KA, Wenger AM, Berger MJ, Guturu H, Stenson PD, Cooper DN, et al. M-CAP eliminates a majority of variants of uncertain significance in clinical exomes at high sensitivity. Nat Genet. 2016;48:1581-6.

25. Samocha KE, Kosmicki JA, Karczewski KJ, O'Donnell-Luria AH, Pierce-Hoffman E, MacArthur DG, et al. Regional missense constraint improves variant deleteriousness prediction. bioRxiv. 148353. https://doi.org/10.1101/148353.

26. Li B, Krishnan VG, Mort ME, Xin F, Kamati KK, Cooper DN, et al. Automated inference of molecular mechanisms of disease from amino acid substitutions. Bioinformatics. 2009:25:2744-50.

27. Qi H, Chen C, Zhang H, Long JJ, Chung WK, Guan Y, et al. MVP: predicting pathogenicity of missense variants by deep learning. bioRxiv. 259390 https://doi.org/10.1101/259390.

28. Sundaram L, Gao H, Padigepati SR, McRae JF, Li Y, Kosmicki JA, et al. Predicting the clinical impact of human mutation with deep neural networks. Nat Genet. 2018:50:1161-70.

29. Ioannidis NM, Rothstein JH, Pejaver V, Middha S, McDonnell SK, Baheti S, et al. REVEL: an ensemble method for predicting the pathogenicity of rare missense variants. Am J Hum Genet. 2016;99:877-85

30. Vaser R, Adusumalli S, Leng SN, Sikic M, Ng PC. SIFT missense predictions for genomes. Nat Protoc. 2016;11:1-9.

31. Balasubramanian S, Fu Y, Pawashe M, McGillivray P, Jin M, Liu J, et al. Using ALoFT to determine the impact of putative loss-of-function variants in protein-coding genes. Nat Commun. 2017:8:382.

32. Siepel A, Pollard KS, Haussler D. New methods for detecting lineage-specific selection. RECOMB 2006 LNCS (LNBI), vol 3909. Heidelberg: Springer; 2006. p. 190-205.

33. Siepel A, Bejerano G, Pedersen JS, Hinrichs AS, Hou M, Rosenbloom K, et al. Evolutionarily conserved elements in vertebrate, insect, worm, and yeast genomes. Genome Res. 2005;15:1034-50.

34. McVicker G, Gordon D, Davis C, Green P. Widespread genomic signatures of natural selection in hominid evolution. PLoS Genet. 2009;5:e1000471.

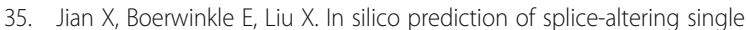
nucleotide variants in the human genome. Nucl Acids Res. 2014;42:13534-44.

36. Xiong HY, Alipanahi $B$, Lee $L$, Bretschneider $H$, Merico D, Yuen RKC, et al. RNA splicing. The human splicing code reveals new insights into the genetic determinants of disease. Science. 2015;347:1254806.

37. Jaganathan K, Kyriazopoulou Panagiotopoulou S, McRae JF, Darbandi SF, Knowles D, Li Yl, et al. Predicting splicing from primary sequence with deep learning. Cell. 2019;176:535-548.e24

38. Li C, Mou C, Swartz MD, Yu B, Bai Y, Tu Y, et al. dbMTS: a comprehensive database of putative human microRNA target site SNVs and their functional predictions. Hum Mutat. 2020:41:1123-30.

39. Hinrichs AS, Karolchik D, Baertsch R, Barber GP, Bejerano G, Clawson H, et al. The UCSC genome browser database: update 2006. Nucleic Acids Res. 2006; 34:D590-8.

40. The UniProt Consortium. Reorganizing the protein space at the Universal Protein Resource (UniProt). Nucleic Acids Res. 2011·40:D71-5.

41. Jm R, P M, I E, A P, Jj W, G L, et al. APPRIS: annotation of principal and alternative splice isoforms. Nucleic Acids Res 2012;41:D110-D117.

42. Guberman JM, Ai J, Arnaiz O, Baran J, Blake A, Baldock R, et al. BioMart Central Portal: an open database network for the biological community. Database (Oxford). 2011;2011:bar041.

43. Liu X, White $S$, Peng $B$, Johnson AD, Brody JA, Li AH, et al. WGSA: an annotation pipeline for human genome sequencing studies. J Med Genet. 2016:53:111-2.

44. Shihab HA, Gough J, Cooper DN, Stenson PD, Barker GLA, Edwards KJ, et al. Predicting the functional, molecular, and phenotypic consequences of amino acid substitutions using hidden Markov models. Hum Mutat. 2013:34:57-65.

45. Reva B, Antipin Y, Sander C. Predicting the functional impact of protein mutations: application to cancer genomics. Nucleic Acids Res. 2011:39:e118.

46. Adzhubei IA, Schmidt S, Peshkin L, Ramensky VE, Gerasimova A, Bork P, et al. A method and server for predicting damaging missense mutations. Nat Methods. 2010;7:248-9.

47. Choi Y, Sims GE, Murphy S, Miller JR, Chan AP. Predicting the functional effect of amino acid substitutions and indels. PLOS One. 2012:7:e46688.

48. Ng PC, Henikoff S. Predicting deleterious amino acid substitutions. Genome Res. 2001:11:863-74. 
49. Carter H, Douville C, Stenson PD, Cooper DN, Karchin R. Identifying Mendelian disease genes with the variant effect scoring tool. BMC Genomics. 2013;14(Suppl 3):S3.

50. Chun S, Fay JC. Identification of deleterious mutations within three human genomes. Genome Res. 2009;19:1553-61.

51. Schwarz JM, Cooper DN, Schuelke M, Seelow D. MutationTaster2: mutation prediction for the deep-sequencing age. Nat Methods. 2014;11:361-2.

52. Dong C, Wei P, Jian X, Gibbs R, Boerwinkle E, Wang K, et al. Comparison and integration of deleteriousness prediction methods for nonsynonymous SNVs in whole exome sequencing studies. Hum Mol Genet. 2015;24:2125-37.

53. Shihab HA, Rogers MF, Gough J, Mort M, Cooper DN, Day INM, et al. An integrative approach to predicting the functional effects of non-coding and coding sequence variation. Bioinformatics. 2015;31:1536-43.

54. Liu X, Li C, Boerwinkle E. The performance of deleteriousness prediction scores for rare non-protein-changing single nucleotide variants in human genes. J Med Genet. 2017;54:134-44.

\section{Publisher's Note}

Springer Nature remains neutral with regard to jurisdictional claims in published maps and institutional affiliations.

Ready to submit your research? Choose BMC and benefit from:

- fast, convenient online submission

- thorough peer review by experienced researchers in your field

- rapid publication on acceptance

- support for research data, including large and complex data types

- gold Open Access which fosters wider collaboration and increased citations

- maximum visibility for your research: over $100 \mathrm{M}$ website views per year

At BMC, research is always in progress.

Learn more biomedcentral.com/submissions 